\title{
Diarrhées persistantes dans les pays en développement
}

\author{
Zulfiqar A. Bhutta \\ Service de Pédiatrie et de Santé de l'Enfant, Université Aga Khan, Karachi, Pakistan
}

\author{
Mots-clés \\ Diarrhée persistante · Carence en micronutriments · \\ Malnutrition - Immunodéficience · Traitement \\ inapproprié, diarrhée aiguë
}

\section{Résumé}

Les affections diarrhéiques sont globalement responsables d'un cinquième de la mortalité totale des enfants de moins de cinq ans, avec un nombre annuel de décès estimé à 2.2 millions. Une proportion significative de ces décès peut être liée à des épisodes de diarrhée persistante (définie comme une diarrhée persistant plus de 15 jours avec altération de la croissance). II a été démontré que le cadre des diarrhées persistantes rassemblait des enfants chez lesquels la diarrhée était nettement plus sévère. Ces affections sont à l'origine de 36 à $54 \%$ de la mortalité totale due à des diarrhées. Bien que la pathogénie exacte de la diarrhée persistante demeure mal élucidée, la plupart des épisodes sont liés à des infections diarrhéiques aiguës antérieures avec prolongation de l'épisode le plus récent. Cette situation peut être liée à plusieurs facteurs propres à l'hôte tel que la préexistence $d^{\prime}$ 'une malnutrition, d'une carence en micronutriments et d'une immunodéficience et le traitement inadéquat de l'épisode le plus récent de diarrhée aiguë. La conséquence commune paraît être des lésions persistantes de la muqueuse intestinale qui ne parvient pas à se ré- parer. Contrairement aux diarrhées persistantes post-infectieuses, certains nourrissons peuvent présenter une diarrhée intraitable sévère due à divers troubles, dont beaucoup ont une base héréditaire. Ils forment aujourd'hui le type dominant de diarrhée prolongée dans les pays industrialisés.

La prise en charge d'une diarrhée persistante repose sur une grande vigilance en matière de renutrition et sur I'identification précoce de complications. Bien que divers germes pathogènes aient été associés aux diarrhées persistantes, la place des médicaments antimicrobiens oraux est restreinte dans le traitement de routine de ces affections. L'attention portée à la réhydratation, le dépistage et le traitement appropriés des infections systémiques et la renutrition par la nutrition entérale au moyen d'aliments facilement absorbables sont essentiels pour la prise en charge de ces enfants, fréquemment dans un contexte ambulatoire. L'administration de zinc et de vitamine A peut raccourcir la durée d'une diarrhée persistante et aide également à réduire les récidives. Les stratégies les plus importantes de prévention de ces diarrhées demeurent cependant I'allaitement maternel exclusif au cours des six premiers mois de la vie, poursuivi durant deux ans avec des aliments complémentaires adéquats et une prise en charge optimale des épisodes diarrhéiques aigus.

Copyright (c) 2006 Nestec Ltd., Vevey/S. Karger AG, Basel

\section{KARGER}

Fax +4161306 1234 E-Mail karger@karger.ch www.karger.com
C) 2006 Nestec Ltd. Vevey/S. Karger AG, Basel 0250-9644/06/0641-0039\$23.50/0

Accessible en ligne à: www.karger.com/anf
Zulfiqar Ahmed Bhutta

Department of Paediatrics and Child Health, Aga Khan University PO Box 3500, Stadium Road

Karachi 74800 (Pakistan)

Tel. +92 21 4930051/ext 4724, Fax +92 21 4934294, E-Mail zulfiqar.bhutta@aku.edu 


\section{Introduction}

Malgré les progrès considérables des connaissances et de la prise en charge des affections diarrhéiques infantiles, celles-ci demeurent globalement responsables d'une importante mortalité infantile (20\%), avec un nombre annuel de décès estimé à 2.2 millions [1]. Dans le cadre d'une estimation du poids des affections diarrhéiques en 1980, l'Organisation Mondiale de la Santé a calculé, que plus de 700 millions d'épisodes de diarrhée affectaient chaque année les enfants de moins de 5 ans dans les pays en développement (à l'exception de la Chine) et étaient responsables d'environ 4.6 millions de décès [2]. Des analyses plus récentes des études publiées ces dix dernières années indiquent que, bien que la mortalité globale ait diminué, l'incidence globale demeurait inchangée, à environ 3.2 épisodes par enfant-année [2-5]. Il est toutefois important de souligner que le nombre d'enquêtes épidémiologiques de bonne qualité entre ces périodes a également significativement varié et que les études récemment menées sont relativement peu nombreuses. L'enquête entreprise en 1993 a inclus 276 études menées dans 60 pays [3], alors que l'analyse de 2003 n'a porté que sur les données de 31 études réalisées dans 20 pays [5].

Il est reconnu que la plupart des épisodes diarrhéiques forment un continuum, les troubles disparaissant en moins de sept jours dans la majorité des cas. Une petite proportion des épisodes diarrhéiques persiste cependant pendant plus de deux semaines. Une diarrhée persistante se définit par un épisode qui débute de façon aiguë mais qui dure au moins 15 jours [6]. Ces critères caractérisent des enfants chez lesquels la diarrhée est nettement plus importante et l'incidence des décès dus à la diarrhée plus élevée. La proportion de ces épisodes a été estimée à 3 à $20 \%$ de l'ensemble des épisodes diarrhéiques chez les enfants de moins de cinq ans [7]. Plusieurs importantes études des diarrhées dans le contexte communautaire $[8,9]$ ont montré que les diarrhées persistantes sont directement responsables de 36 à $45 \%$ de la mortalité totale due à ces affections. Il a été cependant souligné que ces différences de mortalité entre des régions différentes ont pu être liées à des caractéristiques environnementales et à des différences d'utilisation des ressources de santé et de la réhydratation orale [10]. Les diarrhées persistantes n'ont représenté que 5\% des épisodes diarrhéiques lors d'une importante étude prospective des diarrhées en Inde du nord, mais ont été responsables de 14\% de la mortalité, contre $0,7 \%$ pour les épisodes de plus courte durée [11].
Il est important de noter que la plupart des diarrhées persistantes survenant dans les pays en développement sont post-infectieuses. Dans ces pays, la majorité des diarrhées débute de façon aiguë et dure moins d'une semaine. La durée de ces épisodes est cependant plus longue, excédant parfois 15 jours chez certains enfants [12]. Bien que la classification susmentionnée des épisodes diarrhéiques en diarrhées aigues et diarrhées persistantes puisse être arbitraire, elle correspond à une définition opérationnelle identifiant les enfants chez lesquels l'atteinte diarrhéique est significativement plus élevée. Une étude prospective menée dans le nord-est du Brésil par Lima et coll. [13] a montré que tous les enfants chez lesquels l'atteinte diarrhéique avait été importante (présence d'une diarrhée pendant plus de $16 \%$ du nombre total de jours) avaient présenté au moins un épisode de diarrhée persistante en une année d'étude. Des observations similaires de Moy et coll. [14] dans une cohorte de 204 enfants vivant en milieu rural au Zimbabwe ont montré que les diarrhées persistantes n'ont représenté que $6 \%$ des épisodes diarrhéiques, mais $22 \%$ des jours totaux de diarrhée enregistrés lors de cette étude, et que les récidives diarrhéiques avaient été également plus fréquentes lors du suivi chez ces enfants. Les enfants atteints d'une diarrhée persistante peuvent donc ne pas représenter un sous-groupe distinct mais faire partie d'un continuum de sensibilité à la diarrhée. De nombreux enfants des pays en développement peuvent présenter de fréquents épisodes récidivants de diarrhée aiguë, particulièrement au cours des deux premières années de leur vie. La durée des épisodes individuels aigus peut être le plus souvent inférieure à 15 jours, mais la fréquence de leurs récidives peut altérer la situation nutritionnelle et prédisposer ces enfants à une diarrhée persistante.

Contrairement à celles survenant dans les pays en développement, les diarrhées persistantes affectant les jeunes enfants des pays industrialisés se situent dans la catégorie des diarrhées infantiles intraitables. Ces cas se définissent par une diarrhée persistant pendant au moins deux semaines et pouvant nécessiter l'apport par voie intraveineuse d'au moins $50 \%$ des besoins liquidiens quotidiens. Nombre de ces troubles ont une base génétique et peuvent également être observés dans les pays en développement, bien que leur prévalence soit éclipsée par celle des diarrhées persistantes post-infectieuses. 


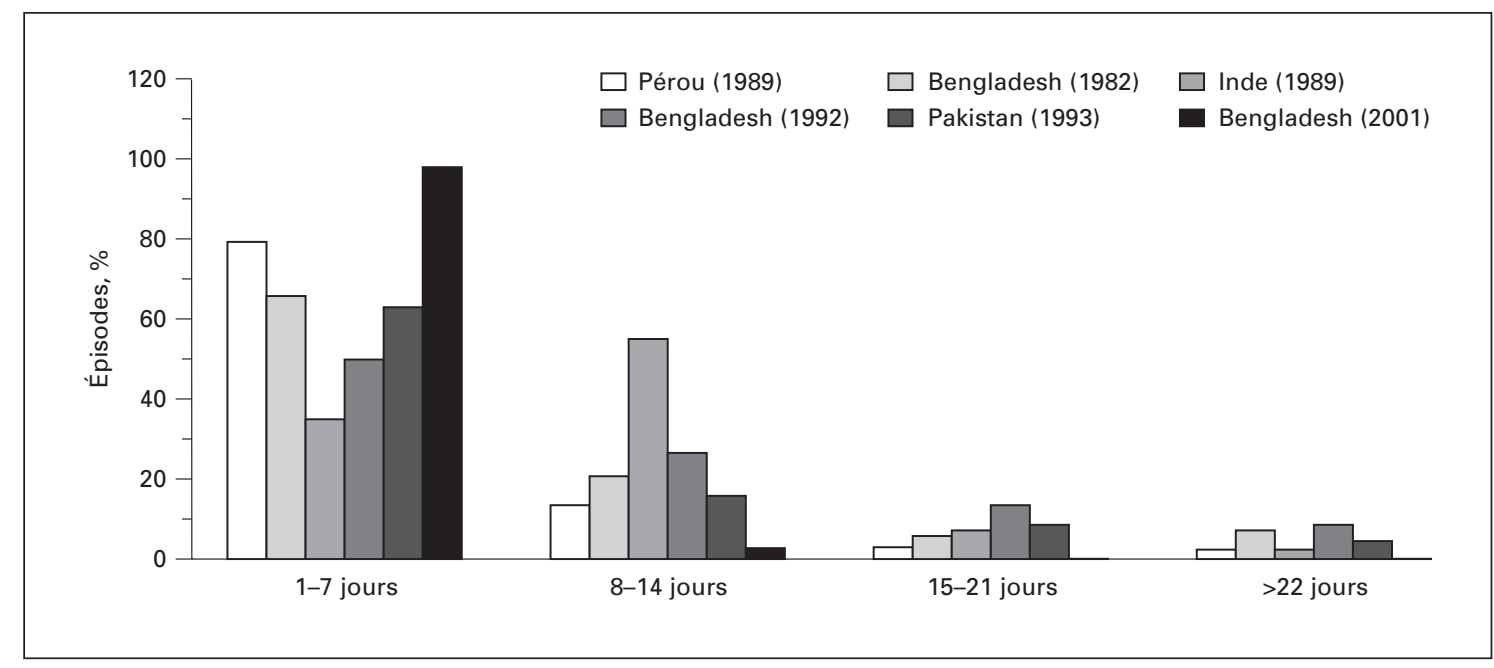

Fig. 1. Incidence des diarrhées infantiles dans des contextes communautaires [11, 17-20].

\section{Méthodes d'analyse}

Un groupe de travail sur les diarrhées persistantes a récemment analysé les informations et les actions nécessaires à l'échelon des praticiens, des organismes de formation et des sociétés professionnelles [15]. Cette analyse n'a cependant pas porté sur les tendances épidémiologiques récentes ni sur les interventions nécessaires dans les systèmes de santé nationaux. Notre propre analyse présentera un aperçu de l'état actuel des informations épidémiologiques et des données manquantes en matière de soins cliniques ainsi que d'interventions de santé publique.

Afin d'évaluer les informations et tendances devenues récemment disponibles, nous avons analysé toutes les données publiées ces 15 dernières années (1990-2004) sur l'épidémiologie des diarrhées et répertoriées par des bibliothèques électroniques médicales (PubMed/Medline, Extra Med, Pop Line) et de sciences sociales. Nous avons également utilisé des termes tel que «diarrhée chronique», «diarrhée prolongée» et «diarrhée intraitable» (en langue anglaise) afin d'effectuer des recherches dans les publications pertinentes. De plus, nous avons réalisé une recherche manuelle dans des monographies adéquates et des documents de l'OMS/UNICEF afin d'obtenir des informations récentes sur les diarrhées persistantes et les stratégies de traitement concernant l'enfant. Nous avons alors classé les études disponibles dans les catégories suivantes : estimations de l'épidémiologie ou du poids de ces affections, pathogenie, stratégies thérapeuti- ques en établissement de soins ou dans la communauté et essais randomisés et contrôlés. Nous avons également évalué les études des diarrhées persistantes ayant trait au statut de populations vis-à-vis du VIH et d'agents pathogènes tel que cryptosporidies, Giardia lamblia, etc.

La plus grande partie des informations épidémiologiques sur la relation entre épisodes diarrhéiques aigus et diarrhée persistante a été obtenue à partir d'études menées voici plus d'une décennie. Les données récentes en ce domaine ont été remarquablement peu nombreuses, particulièrement concernant les zones où l'infection à VIH n'est pas endémique. Les études menées dans les zones d'endémie de cette infection ont toutefois nettement montré que les entéropathies chroniques et les diarrhées persistantes étaient de plus en plus reconnues comme des manifestations fréquentes d'une infection avancée à VIH et d'un sida [16].

Dans l'ensemble, peu de données montrent que l'incidence globale réelle des diarrhées persistantes a diminué. La figure 1 indique la durée des diarrhées observée lors de plusieurs études communautaires menées dans des pays en développement [11, 17-20]. Les données issues d'études communautaires récentes sur les diarrhées sont relativement rares, mais l'incidence globale des diarrhées persistantes dans ce contexte paraît remarquablement constante de 1980 à 1992. Une récente étude de ce type menée au Bangladesh [20] indique que les diarrhées persistantes ont représenté moins de $0.5 \%$ de l'ensemble des cas de diarrhée dans une communauté urbaine, mais il est difficile d'effectuer des généralisations concernant une 
Tableau 1. Agents pathogènes associés à des diarrhées prolongées et persistantes

\begin{tabular}{ll}
\hline Bactéries & Escherichia coli entéroadhérent \\
& Escherichia coli entéropathogène \\
& Campylobacter \\
& Salmonella enteritides \\
& Shigella \\
& Clostridium difficile \\
& Arcobacter butzleri \\
& Klebsiella \\
\hline Parasites & Giardia lamblia \\
& Blastocystis hominis ${ }^{1}$ \\
& Cryptosporidium ${ }^{1}$ \\
& Entamoeba histolytica \\
& Cyclospora cayetanensis ${ }^{1}$ \\
\hline Virus & Astrovirus humain 3 \\
& Entérovirus \\
& Picobirnavirus \\
\hline
\end{tabular}

${ }^{1}$ En particulier en association à une infection au VIH/sida.

Tableau 2. Étiologie des diarrhées infantiles intraitables

1 Diarrhée chlorée congénitale

2 Maladie des inclusions microvillositaires.

3 Dysplasie épithéliale intestinale ou tufting entéropathy

4 Entéropathie auto-immune

5 Allergie alimentaire

6 Pseudo-occlusion intestinale chronique/maladie de Hirschsprung

7 Lymphangiectasies intestinales

8 Abêtalipoprotéinémie

9 Entéropathie à éosinophiles

10 Neurodysplasie intestinale

11 Déficit enzymatique congénital (malabsorption congénitale du glucose et du galactose, déficit en saccharase-isomaltase, etc.)

12 Trichorrhexie noueuse, dysmorphie faciale, immunodéficience et cirrhose

tendance à une réduction de la charge due à ces formes persistantes. Il est manifestement nécessaire de revoir les proportions précédemment estimées des épisodes diarrhéiques aigus qui se prolongent.

L'épidémie de VIH/sida en Afrique a modifié le profil étiologique habituel des diarrhées. Les épisodes prolongés associés à des infections par des germes relativement rares tels que les cryptosporidies sont plus fréquents.

\section{Pathogenèse des diarrhées persistantes}

Les diarrhées infantiles prolongées peuvent également être secondaires à un grand nombre d'affections dont beaucoup ne sont pas infectieuses. Les plus fréquentes sont l'intolérance au protéines du lait de vache ou de soja (chez les nourrissons de moins de 6 mois, souvent avec des selles striées de sang), la maladie coeliaque (entéropathie sensible au gluten) et la mucoviscidose (créatorrhée et stéatorrhée secondaires à une insuffisance pancréatique). Dans d'autres cas, une diarrhée peut également survenir chez des nourrissons relativement bien nourris.

Toutefois, le facteur déclenchant le plus important de diarrhée persistante est globalement un épisode diarrhéique aigu dû à une infection intestinale et aux conséquences de celle-ci. Plusieurs études ont été menées sur les agents pathogènes responsables de diarrhées persistantes dans les pays en développement, mais peu ont démontré la responsabilité majeure de l'un d'entre eux. Les diarrhées persistantes peuvent apparaître comme une conséquence d'une prise en charge inadéquate de diarrhées infectieuses aiguës, mais certains agents pathogènes sont plus fréquemment isolés chez les enfants qu'elles atteignent. Le tableau 1 indique les divers germes qui ont été associés aux diarrhées persistantes chez des enfants de diverses parties du monde.

Globalement, près de $25 \%$ des décès dus aux diarrhées sont associés à des dysenteries dont une grande partie est due à des shigelles. Une récente étude menée au Bengladesh [21] a montré qu'une diarrhée persistante était présente chez 23\% des enfants atteints d'une shigellose, et que les infections à Shigella étaient significativement associées à une augmentation du risque de prolongation de la diarrhée (risque relatif corrigé en fonction de l'âge: 1.83; intervalle de confiance à 95\% 1.19-2.81). Une infection par une souche de Shigella multirésistante (risque relatif corrigé en fonction de l'âge : 3.76 ; intervalle de confiance à 95\% 1.51-9.36) et la survenue d'une shigellose au cours de la première année de la vie sont des facteurs de risque de persistance d'une diarrhée à Shigella. Toutefois, $88 \%$ des épisodes persistants de shigellose sont survenus chez des enfants plus âgés, $50 \%$ ont été associés à une shigellose non dysentérique et $79 \%$ ont été dus à des espèces de Shigella autres que Shigella dysenteriae. D'autres germes peuvent induire une inflammation muqueuse similaire. Un mécanisme pathogénique supplémentaire sous-tendant les infections à Escherichia coli entéroadhérent peut induire l'IL-8 et une inflammation muqueuse. 


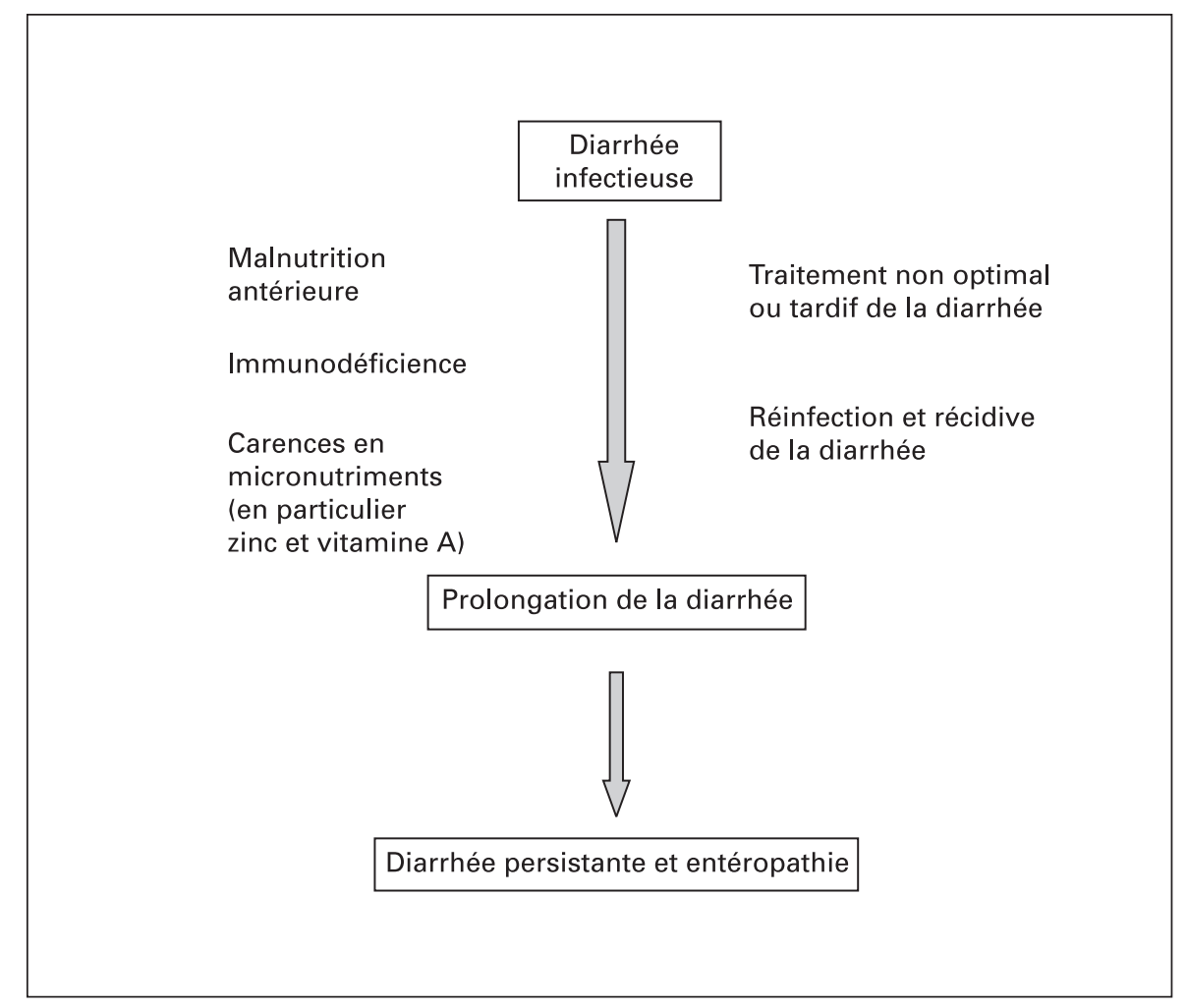

Fig. 2. Constitution progressive d'une diarrhée prolongée et persistante.

Les mécanismes sous-jacents d'absorption et de transport muqueux sont beaucoup mieux connus dans le cas des diarrhées intraitables survenant dans les pays industrialisés que dans celui des diarrhées persistantes. Le tableau 2 présente certaines des principales causes de diarrhée infantile intraitable.

On sait aujourd'hui que des mutations de l'échangeur $\mathrm{Cl}^{-} / \mathrm{HCO}_{3}^{-}$de la membrane apicale iléocolique (famille 26 de transporteurs solubles, gène numéro 3 [SLC26A3, encore appelé CLD ou DRA]) entaînent la sécrétion nette de chlorures par l'intestin et un syndrome chronique appelé diarrhée chlorée congénitale [22]. On sait de même que le syndrome de malabsorption congénitale du glucose et du galactose est dû à une anomalie génétique résultant d'une réduction ou d'une absence du transporteur fonctionnel du glucose dépendant du sodium (SGLT1) a la surface des entérocytes [23], et diverses relations génotype/phénotype ont été identifiées [24]. Un déficit congénital en saccharase-isomaltase se manifeste également par la survenue d'une diarrhée osmotique à la suite de l'introduction d'aliments de sevrage contenant des amidons complexes ou du saccharose. Des études biologiques moléculaires et cellulaires ont extrêmement bien défini la pathogénie de ce déficit en démontrant qu'il paraissait résulter à la fois d'une anomalie de synthèse et de maturation de la glycoprotéine [25].

En revanche, les études approfondies menées dans des pays en développement sur les anomalies de la muqueuse et les facteurs sous-tendant celles-ci dans les diarrhées persistantes sont malheureusement rares, et aucune n'a exploré les mécanismes moléculaires d'une atteinte intestinale prolongée. L'exemple le plus frappant du rôle capital du système immunitaire dans la pathogenie des diarrhées persistantes est la relation entre celles-ci et le VIH/ sida, comme l'ont montré de nombreuses études ayant relié des diarrhées persistantes à une cryptosporidiose [26] et à d'autres parasitoses en Afrique et en Asie. Bien que ces observations suggèrent qu'une immunodéficience pourrait être liée au risque de diarrhée prolongée [27], l'hypothèse selon laquelle un déficit immunitaire transitoire pourrait être aussi un marqueur de carence concomitante en micronutriments a également suscité un grand intérêt [28, 29].

Un autre facteur de retard de réparation des lésions intestinales pourrait être une carence en micronutriments majeurs influençant ce processus. Des études initiales avaient indiqué le bénéfice potentiel d'une supplémentation en zinc pour la réduction du risque de diarrhée pro- 
longée [30] et des méta-analyses récentes montrent que ce traitement réduit significativement la durée et la sévérité d'affections diarrhéiques [31]. Une carence en zinc peut donc significativement contribuer à la persistance des lésions muqueuses et à la lenteur de la cicatrisation intestinale. Les effets bénéfiques du zinc en termes de réduction de la mortalité due aux diarrhées infantiles ont motivé son inclusion dans les recommandations actuelles de l'OMS/UNICEF concernant la prise en charge des diarrhées [32]. Les mécanismes d'action du zinc dans la réduction de la durée et de la sévérité de la diarrhée et le maintien de l'intégrité de la muqueuse intestinale demeurent toutefois mal connus.

Une cicatrisation intestinale inadéquate est considérée comme une cause majeure d'anomalies morphologiques de la muqueuse. Les facteurs précis sous-tendant l'inefficacité des processus de cicatrisation et la persistance des lésions sont toutefois mal compris. Le résultat final des perturbations de la muqueuse est une malabsorption des nutriments et une augmentation de la perméabilité de l'intestin aux antigènes alimentaires ou microbiens. La diarrhée persistante est largement reconnue comme étant un trouble nutritionnel, mais les mécanismes qui la sous-tendent et l'immunopathologie de la muqueuse sont remarquablement mal connus chez les enfants qui présentent ce syndrome. Plusieurs facteurs contribuant à la persistance d'une diarrhée ont été toutefois identifiés, dont une malnutrition générale, des carences en micronutriments et une immunodéficience, ce qui suggère qu'un retard de cicatrisation intestinale à la suite des lésions initiales peut être un important facteur contributif. La figure 2 indique les mécanismes associés à un retard de cicatrisation intestinale et à une entéropathie prolongée pouvant sous-tendre les diarrhées persistantes post-infectieuses.

Il est essentiel de bien connaître les altérations de la morphologie et de la physiologie intestinales afin de pouvoir développer des stratégies d'intervention. Toutefois, des modifications pathologiques très diverses après des diarrhées persistantes ont été décrites dans des études plutôt petites [33-35] rapportant d'importantes observations qui évoquent une inflammation chronique et une altération de l'intégrité de la muqueuse. A cet égard, des travaux beaucoup plus concertés sur des populations représentatives sont nécessaires.

\section{Prise en charge des diarrhées persistantes}

L'aspect le plus important de la prise en charge d'un enfant présentant une diarrhée persistante est l'évaluation immédiate et le tri pour un traitement consécutif. La prise en charge des diarrhées persistantes des enfants malnourris repose sur les principes de celle d'une diarrhée et d'une malnutrition. Bien qu'un sous-groupe puisse présenter une malnutrition sévère nécessitant une renutrition rapide, souvent en milieu hospitalier, une prise en charge ambulatoire peut être possible dans les autres cas. Compte tenu de la longue durée que peut demander la convalescence, une hospitalisation prolongée peut poser d'importants problèmes dans les pays en développement et, chaque fois que possible, un traitement ambulatoire ou à domicile doit être préféré.

Nous indiquons ci-dessous les principes fondamentaux de la prise en charge des diarrhées persistantes, et la figure 3 résume l'approche thérapeutique proposée [36].

\section{(1) Réanimation et stabilisation initiales}

La plupart des enfants présentant une diarrhée persistante et une malnutrition associée ne sont pas sévèrement déshydratés et une réhydratation orale peut donc être adaptée. Des poussées aiguës et des vomissements associés peuvent cependant nécessiter de courtes périodes de réhydratation intraveineuse par Ringer-lactate. Il peut être nécessaire de corriger des déséquilibres électrolytiques aigus tels qu'une hypokaliémie et une acidose sévère. Point plus important, une translocation bactérienne [37] et des infections systémiques ont été identifiées chez des enfants sévèrement dénutris atteints d'une diarrhée persistante et sont une cause fréquente de mortalité précoce. Elles doivent être dépistées dès l'admission. Jusqu'à $50 \%$ des enfants malnourris atteints d'une diarrhée persistante peuvent présenter une infection systémique associée nécessitant une réanimation et une antibiothérapie $[38,39]$. Chez l'enfant sévèrement malnourri nécessitant une hospitalisation, une antibiothérapie à large spectre lors de l'hospitalisation et de l'admission initiales peut être nécessaire dans l'attente du résultat des cultures. Le traitement présomptif d'une cryptosporidiose peut être également nécessaire dans certains cas, particulièrement chez les enfants infectés par le VIH [40].

\section{(2) Réhydratation orale}

La solution standard de réhydratation orale de l'OMS est généralement adaptée pour compenser les pertes liquidiennes en cours, mais des données récentes indiquent que des liquides de réhydratation orale de basse osmola- 


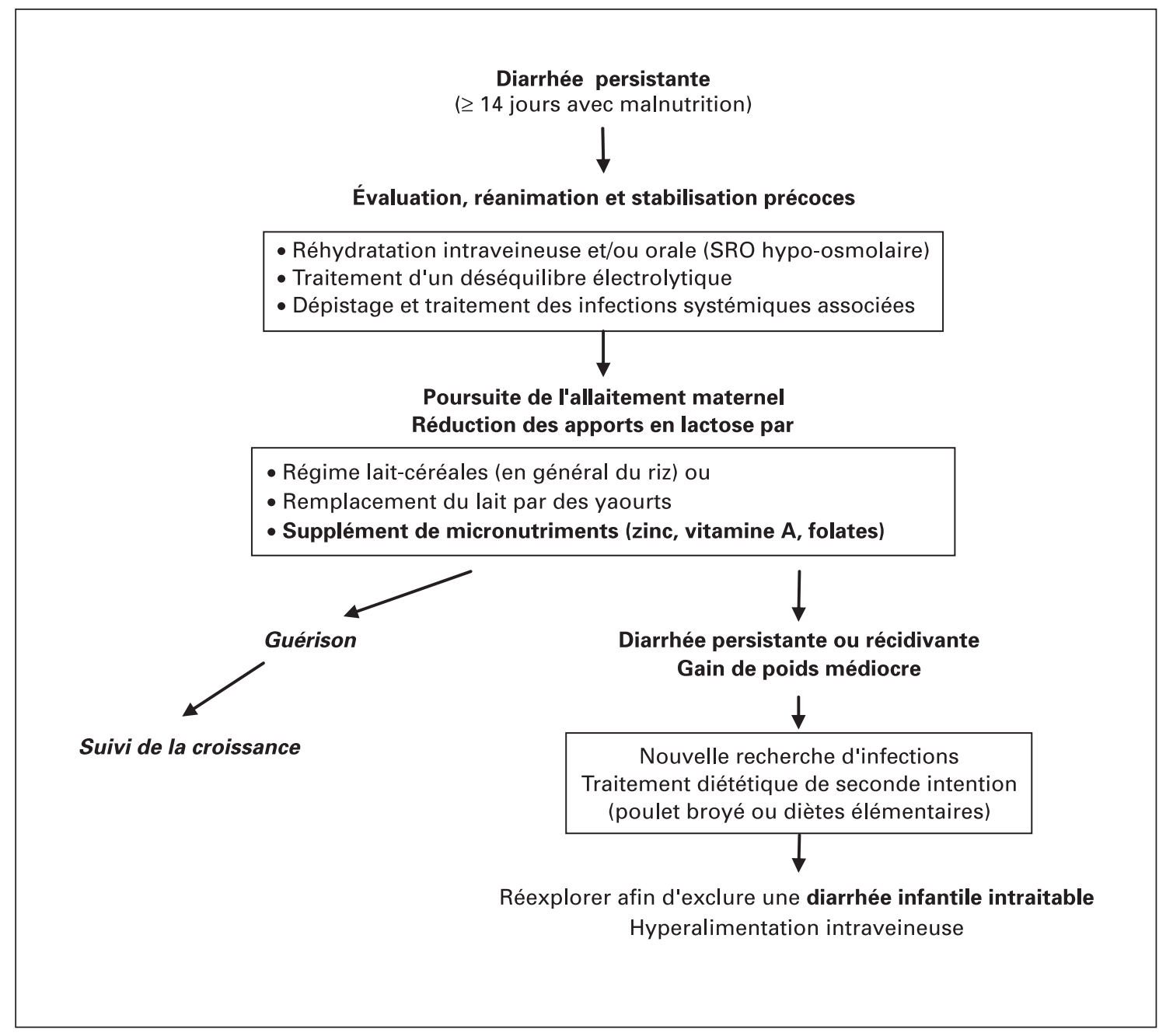

Fig. 3. Algorithme suggéré de prise en charge d'une diarrhée persistante.

lité $[41,42]$ peuvent être avantageux chez les nourrissons malnourris sans les exposer au risque d'hyponatrémie.

\section{(3) Nutrition entérale et sélection de la diète}

Il est extrêmement rare qu'une diarrhée persistante survienne chez un nourrisson sous allaitement maternel exclusif et, à l'exception de contextes spécifiques, où la persistance de la diarrhée accompagne une infection à VIH contractée en période périnatale, l'allaitement maternel doit être poursuivi.

Malgré les anomalies de la muqueuse et l'altération des mécanismes de digestion et d'absorption, les capacités d'absorption et de tolérance des aliments entéraux est adéquate chez la plupart des enfants atteints d'une diarrhée persistante. Des études du bilan métabolique chez des enfants malnourris présentant une diarrhée persistante indiquent que l'absorption des glucides, des protéines et des lipides peut être adéquate sous divers régimes alimentaires [43, 44]. L'arrêt du lait et son remplacement par des formules sans lactose spécialisées (et coûteuses) est donc généralement inutile.

La plupart des enfants atteints d'une diarrhée persistante ne sont pas intolérants au lactose, bien que l'administration de ce sucre à raison de plus de $5 \mathrm{~g} / \mathrm{kg} /$ jour soit associée à une augmentation du débit fécal et à des échecs thérapeutiques [45]. D'autres stratégies de réduction des apports en lactose chez les enfants malnourris présentant une diarrhée persistante sont l'ajout de lait à des céréales et le remplacement du lait par des produits laitiers fermentés tels que le yaourt. Plusieurs études ont évalué ces 
interventions diététiques de façon approfondies et ont montré qu'elles étaient efficaces et acceptables par rapport à des formules coûteuses $[7,46]$.

Dans de rares cas, quand une intolérance alimentaire interdit l'administration de lait de vache ou de formules à base de celui-ci, un régime alimentaire non lacté spécialisé, par exemple à base de poulet broyé ou mixé, ou une formule élémentaire peut être nécessaire [47]. Il faut souligner que, bien qu'efficace dans certains contextes [48], ce type de formules est trop coûteux pour la plupart des pays en développement. Une association de poulet broyé et de gomme guar s'est avérée efficace chez des enfants présentant une diarrhée persistante [49], et il en a été de même de l'ajout de bananes vertes ou de pectine à la diète $[50]$.

La densité énergétique habituelle de tout régime alimentaire utilisé pour le traitement d'une diarrhée persistante doit être voisine de $1 \mathrm{kcal} / \mathrm{g}$ afin d'assurer des apports quotidiens d'au moins $100 \mathrm{kcal} / \mathrm{kg}$ d'énergie et de 2 à $3 \mathrm{~g} / \mathrm{kg}$ de protéines. Les préparations de riz et lentilles communément utilisées en Asie du sud tel que le khitchri apportent cette densité énergétique en association à un apport proteique et une composition en acides aminés optimaux.

\section{(4) Supplémentation en micronutriments}

Il est largement reconnu que des carences associées en micronutriments, dont le zinc, le fer et la vitamine A, existent chez la plupart des enfants malnourris atteints d'une diarrhée persistante. Elles peuvent être la conséquence d'apports insuffisants et de pertes intestinales persistantes et nécessitent une supplémentation au cours du traitement [51]. Les données en faveur de l'administration de zinc chez les enfants présentant une diarrhée persistante sont convaincantes, mais il est probable que ceux-ci souffrent de carences en plusieurs micronutriments. Des études ont montré que l'administration concomitante de vitamine A chez des enfants atteints d'une diarrhée persistante améliorait l'évolution [52, 53], particulièrement dans les zones où l'infection à VIH est endémique [54]. Il est donc important d'assurer que tous les enfants malnourris présentant une diarrhée persistante reçoivent une dose initiale de 100000 unités de vitamine A et au moins $3-5 \mathrm{mg} / \mathrm{kg} / \mathrm{jour}$ de zinc élément.

\section{(5) Suivi et rééducation nutritionnelle dans les contextes communautaires}

En raison de la fréquence élevée des récidives chez la plupart des enfants atteints d'une diarrhée persistante, il est important de prendre en charge les facteurs de risque et d'instaurer des mesures préventives, dont une alimentation adéquate (allaitement maternel et aliments de complément) une étroite attention à l'hygiène et aux conditions sanitaires de l'environnement, ce qui pose des problèmes considérables dans les communautés dépourvues des nécessités de base telle qu'une eau non contaminée et des dispositifs d'élimination des déchets.

Outre celles ayant trait aux aspects préventifs, les difficultés rencontrées dans la plupart des contextes concernent la réalisation et le maintien d'une forme de traitement diététique des diarrhées persistantes de l'enfant utilisant des ingrédients peu coûteux, disponibles à domicile et culturellement acceptables. Considérant que la majorité des cas de diarrhée persistante surviennent dans la communauté et que les parents hésitent fréquemment à consulter une institution, il est nécessaire de développer et de mettre en œuvre des mesures thérapeutiques peu coûteuses et pratiques applicables à domicile. Les données disponibles indiquent qu'il peut être tout à fait possible d'agir ainsi dans des contextes communautaires $[55,56]$.

\section{Stratégies de prévention des diarrhées persistantes}

Bien qu'une renutrition optimale des enfants atteints d'une diarrhée persistante soit considérée comme la clé de voûte du traitement [44], la prévention est capitale. A cette fin, des améliorations à grande échelle des stratégies de prévention et de prise en charge des diarrhées dans les pays en développement sont essentielles. Comme nous l'avons indiqué dans une analyse des interventions possibles afin d'améliorer la survie des enfants, celles-ci peuvent prévenir 55\% de l'ensemble des décès chez les enfants de moins de 5 ans [57], dont un cinquième est liée à une diarrhée.

Ces interventions incluent l'allaitement maternel exclusif et des stratégies sûres d'alimentation de complément afin de prévenir les diarrhées et, si un épisode survient malgré tout, d'assurer une prise en charge optimale avec une amélioration de l'hydratation orale, un supplément de zinc et une poursuite de l'alimentation. Comme indiqué plus haut, cette approche exige que tous les épisodes de diarrhée soient rapidement identifiés et traités de façon optimale par réhydratation orale et nutrition entérale par des aliments peu coûteux, disponibles à domicile et culturellement acceptables pouvant être utilisés afin de prendre en charge les diarrhées persistantes infantiles dans le contexte ambulatoire. Les prochaines étapes 
seraient d'intégrer ces interventions afin que les familles et les travailleurs sociaux communautaires puissent les mettre en œuvre dans des situations diverses [58].

Il est nécessaire d'assurer dans les systèmes de santé, les interventions préventives et thérapeutiques essentielles pour réduire les diarrhées persistantes et réaliser ainsi l'un des objectifs de développement du millénaire, la réduction de la mortalité infantile des deux tiers d'ici 2015. L'introduction précoce et non hygiénique de laits autres que le lait maternel et le traitement inadéquat des épisodes récidivants de diarrhée aiguë sont d'importants facteurs prédisposant à la survenue de diarrhées persistantes, et il est donc important de les prévenir. Ces facteurs de risque sont généralement fréquents dans les communautés pauvres, et tant la réduction de la pauvreté que les mécanismes de soutien social sont fondamentalement importants. De ce fait, promouvoir l'allaitement maternel pendant au moins 6 mois, éviter une alimentation par des formules non hygiéniques et un sevrage adapté avec des nutriments hygiéniques aideront à prévenir les épiso- des de diarrhée persistante post-infectieuse. Ces méthodes doivent être couplées à des mesures de contrôle de l'environnement et à la fourniture d'eau non contaminée et d'aliments sûrs [59]. Si cela n'est pas possible, des interventions promouvant des méthodes de lavage des mains et des mesures domestiques de purification de l'eau sont capitales pour réduire la charge due aux diarrhées $[60,61]$.

L'affectation de ressources appropriées pour la santé publique et la satisfaction des nécessités de base doivent être associées à la formation des personnels et à la mobilisation de la communauté afin de réduire le poids global des diarrhées et celui de leurs formes persistantes, ce qui nécessite des efforts soutenus et concertés. Dans de nombreux pays en développement, ces actions nécessitent que, au delà des interventions biomédicales, nous attachions une importance capitale à la réduction de la pauvreté et à la promotion d'un programme de respect des droits de l'homme et d'équité.

\section{Références}

-1 Black RE, Morris SS, Bryce J: Where and why are 10 million children dying every year? Lancet 2003;361:2226-2234.

$\checkmark 2$ Snyder JD, Merson MH: The magnitude of the global problem of acute diarrheal disease: a review of active surveillance data. Bull World Heath Organ 1982;60:605-613.

$\checkmark 3$ Bern C, Martines J, de Zoysa I, Glass RI: The magnitude of the global problem of diarrhoeal disease: a ten-year update. Bull World Health Organ 1992;70:705-714.

4 Yusufzai M, Bhutta ZA: Global burden of childhood diarrhea; in Bhutta ZA (ed): Contemporary Issues in Childhood Diarrhea and Malnutrition. Karachi, Oxford University Press, 2000, pp 1-22.

$\checkmark 5$ Kosek M, Bern C, Guerrant RL: The global burden of diarrheal disease as estimated from studies published between 1990 and 2000. Bull World Health Organ 2003;81:197-2004.

6 Anonymous: Persistent diarrhoea in children in developing countries: memorandum from a WHO Meeting. Bull World Health Organ 1988;66:709-717.

7 Anonymous: Evaluation of an algorithm for the treatment of persistent diarrhoea: a multicentre study. International Working Group on Persistent Diarrhoea. Bull World Health Organ 1996;74:479-489.

8 Schorling JB, Wanke CA, Schorlling SK, McAullife JF, de Souza MA, Guerran RL: A prospective study of persistent diarrhea among children in an urban Brazilian slum: patterns of occurrence and etiologic agents. Am J Epidemiol 1990;132:144-156.
$>9$ Fauveau V, Henry FJ, Briend A, Yunus M, Chakraborty J: Persistent diarrhea as a cause of childhood mortality in rural Bangladesh. Acta Paediatr Suppl 1992;381:12-14.

10 Victora CG, Huttly SRA, Fuch SC, et al: International differences in clinical patterns of diarrhoeal deaths: a comparison of children from Brazil, Senegal, Bangladesh and India. J Diarrhoeal Dis Res 1993;11:25-29.

11 Bhan MK, Bhandari N, Sazawal S, Clemens J, Raj P: Descriptive epidemiology of persistent diarrhoea among young children in rural northern India. Bull World Health Organ 1989;67:281-288.

12 Black RE: Persistent diarrhea in children of developing countries. Pediatr Infect Dis J 1993; 12:751-761.

13 Lima AAM, Fang G, Schorling JB, Albuquerque L, Mc Aulliffe JA, Mota S, Leite R, Guerrant RL: Persistent diarrhea in northeast Brazil: etiologies and interaction with malnutrition. Acta Paediatr SuppI 1992;381:39-44.

14 Moy RJ, Booth IW, Choto R-G, McNeish AS: Recurrent and persistent diarrhoea in a rural Zimbabwean community: a prospective study. J Trop Pediatr 1991;37:293-299.

15 Bhutta ZA, Ghishan F, Lindley K, Memon IA, Mittal S, Rhoads JM: Persistent and chronic diarrhea and malabsorption: Working Group report of the second World Congress of Pediatric Gastroenterology, Hepatology, and Nutrition. J Pediatr Gastroenterol Nutr 2004; (suppl 2):S711-S716
16 Adejuyigbe EA, Oyelami O, Onayemi O, Durosinmi MA: Paediatric HIV/AIDS in Ile-Ife, Nigeria. Cent Afr J Med 2003;49:74-78.

$\checkmark 17$ Black RE, Brown KH, Becker S, Yunus M: Longitudinal studies of infectious diseases and physical growth of children in rural Bangladesh. I. Patterns of morbidity. Am J Epidemiol 1982;115:305-314.

-18 Lanata CF, Black RE, Gilman RH, Lazo F, Del Aguila R: Epidemiologic, clinical, and laboratory characteristics of acute vs persistent diarrhea in periurban Lima, Peru. J Pediatr Gastroenterol Nutr 1991;12:82-88.

19 Mahmud A, Jalil F, Karlberg J, Lindblad BS: Early child health in Lahore, Pakistan: VII. Diarrhoea. Acta Paediatr Suppl 1993;390:7985 .

20 Haque R, Monda D1, Kirkpatrick BD, Akther Farr BM, Sack RB, Petri WB Jr: Epidemiologic and clinical characteristics of acute diarrhea with emphasis on Entamoeba histolytica infections in preschool children in an urban slum of Dhaka, Bangladesh. Am J Trop Med Hyg 2003;69:398-405.

-21 Ahmed F, Ansaruzzaman M, Haque E, Rao MR, Clemens JD: Epidemiology of postshigellosis persistent diarrhea in young children. Pediatr Infect Dis J 2001;20:525-530.

22 Hoglund P, Haila S, Socha J, Tomaszewski L, Saarialho-Kere U, Karjalainen-Lindsberg ML, Airola K, Holmberg C, de la CA, Kere J: Mutations of the Down-regulated in adenoma (DRA) gene cause congenital chloride diarrhoea. Nat Genet 1996;14:316-319. 
-23 Turk E, Zabel B, Mundlos S, Dyer J, Wright EM: Glucose/galactose malabsorption caused by a defect in the $\mathrm{Na}+$ /glucose cotransporter. Nature 1991;350:354-356.

-24 Martin MG, Turk E, Lostao MP, Kerner C, Wright EM: Defects in Na+/glucose cotransporter (SGLT1) trafficking and function cause glucose-galactose malabsorption. Nat Genet 1996;12:216-220.

-25 Naim HY, Roth J, Sterchi EE, Lentze M, Milla P, Schmitz J, Hauri HP: Sucrase-isomaltase deficiency in humans. Different mutations disrupt intracellular transport, processing, and function of an intestinal brush border enzyme. J Clin Invest 1988;82:667-679.

-26 Amadi B, Kelly P, Mwiya M, Mulwazi E, Sianongo S, Changwe F, Thomson M, Hachungula J, Watuka A, Walker-Smith J, Chintu C: Intestinal and systemic infection, HIV, and mortality in Zambian children with persistent diarrhea and malnutrition. J Pediatr Gastroenterol Nutr 2001;32:550-554.

-27 Baqui A H, Black R E, Sack R B, Chowdhury HR, Yunus M, Siddique A K: Malnutrition, cell-mediated immune deficiency and diarrhea: a community-based longitudinal study in rural Bangladeshi children. Am J Epidemiol 1993; 137:355-365.

-28 Azim T, Ahmad SM, Sefat-E-Khuda, Sarker MS, Unicomb LE, De S, Hamadani JD, Salam MA, Wahed MA, Albert MJ: Immune response of children who develop persistent diarrhea following rotavirus infection. Clin Diagn Lab Immunol 1999;6:690-695.

-29 Taniguchi K, Rikimaru T, Yartey JE, Akpedonu P, Armar-Klemesu MA, Nkrumah FK, Kamiya H, Kishi K, Armar DA: Immunological background in children with persistent diarrhea in Ghana. Pediatr Int 1999;4:162167.

- 30 Sazawal S, Black R E, Bhan M K, Bhandari N, Sinha A, Jalla S: Zinc supplementation in young children with acute diarrhea in India. N Engl J Med 1995;333:839-844.

- 31 Bhutta ZA, Bird SM, Black RE, Brown KH, Gardner JM, Hidayat A, Khatun F, Martorell R, Ninh NX, Penny ME, Rosado JL, Roy SK, Ruel M, Sazawal S, Shankar A: Therapeutic effects of oral zinc in acute and persistent diarrhea in children in developing countries: pooled analysis of randomized controlled trials. Am J Clin Nutr 2000;72:1516-1522.

32 WHO/UNICEF Joint Statement: Clinical Management of Acute Diarrhea. May 2004. http://www.mostproject.org/ZINC/WHOUNICEF/Statement.pdf (accessed Feb 27, 2005).

-33 Sullivan PB: Studies of the small intestine in persistent diarrhea and malnutrition: the Gambian experience. J Pediatr Gastroenterol Nutr 2002;34(suppl 1):S11-S13.

-34 Campbell DI, Murch SH, Elia M, Sullivan PB, Sanyang MS, Jobarteh B, Lunn PG: Chronic T cell-mediated enteropathy in rural west African children: relationship with nutritional status and small bowel function. Pediatr Res 2003;54:306-311.
5 Fagundes-Neto U, De Martini-Costa S, Pedroso MZ, Scaletsky IC: Studies of the small bowel surface by scanning electron microscopy in infants with persistent diarrhea. Braz J Med Biol Res 2000;33:1437-1442.

36 Bhutta ZA: Persistent diarrhea; in Guandalini S (ed): Text Book of Gastroenterology. London, Taylor \& Francis, 2004.

37 Campbell DI, Elia M, Lunn PG: Growth faltering in rural Gambian infants is associated with impaired small intestinal barrier function, leading to endotoxemia and systemic inflammation. J Nutr 2003;133:1332-1338.

38 Bhutta ZA, Nizami SQ, Thobani S: Factors determining recovery during nutritional therapy of persistent diarrhoea: the impact of diarrhoea severity and intercurrent infections. Acta Paediatr 1997;86:796-802.

-39 Alam NH, Faruque AS, Dewan N, Sarker SA, Fuchs GJ: Characteristics of children hospitalized with severe dehydration and persistent diarrhoea in Bangladesh. J Health Popul Nutr 2001;19:18-24.

40 Amadi B, Mwiya M, Musuku J, Watuka A, Sianongo S, Ayoub A, Kelly P: Effect of nitazoxanide on morbidity and mortality in Zambian children with cryptosporidiosis: a randomised controlled trial. Lancet 2002;360: 1375-1380.

41 Sarker SA, Mahalanabis D, Alam NH, Sharmin S, Khan AM, Fuchs GJ: Reduced osmolarity oral rehydration solution for persistent diarrhea in infants: a randomized controlled clinical trial. J Pediatr 2001;138:532-538.

42 Dutta P, Mitra U, Dutta S, Manna B, Chatterjee MK, De A, Bhattacharya SK: Hypo-osmolar oral rehydration salts solution in dehydrating persistent diarrhoea in children: doubleblind, randomized, controlled clinical trial. Acta Paediatr 2000;89:411-416.

43 Bhutta ZA, Molla AM, Isani Z, Badruddin S, Hendricks K, Snyder JD: Dietary management of persistent diarrhea: comparison of a traditional rice-lentil based diet with soy formula. Pediatrics 1991;88:1010-1018.

44 Bhutta ZA, Hendricks KH: Nutritional management of persistent diarrhea in childhood: a perspective from the developing world. J Pediatr Gastroenterol Nutr 1996;22:17-37.

45 Penny ME, Paredes P, Brown KH: Clinical and nutritional consequences of lactose feeding during persistent postenteritis diarrhea. Pediatrics 1989;84:835-844.

46 Ashraf H, Ahmed S, Fuchs GJ, Mahalanabis D: Persistent diarrhoea: associated infection and response to a low lactose diet. J Trop Pediatr 2002;48:142-148.

47 Godard C, Bustos M, Munoz M, Nussle D: Value of a chicken-based formula for refeeding of children with protracted diarrhea and malnutrition in a developing country. J Pediatr Gastroenterol Nutr 1989;9:473-480.

48 Amadi B: Role of food antigen elimination in treating children with persistent diarrhea and malnutrition in Zambia. J Pediatr Gastroenterol Nutr 2002;34(suppl 1):S54-S56.
49 Alam NH, Meier R, Sarker SA, Bardhan PK, Schneider H, Gyr N: Partially hydrolysed guar gum supplemented comminuted chicken diet in persistent diarrhoea: a randomised controlled trial. Arch Dis Child 2005;90:195199.

50 Rabbani GH, Teka T, Zaman B, Majid N, Khatun M, Fuchs GJ: Clinical studies in persistent diarrhea: dietary management with green banana or pectin in Bangladeshi children. Gastroenterology 2001;121:554-560.

51 Mahalanabis D, Bhan MK: Micronutrients as adjunct therapy of acute illness in children: impact on the episode outcome and policy implications of current findings. Br J Nutr 2001; 85(suppl 2):S151-S158.

52 Rahman MM, Vermund SH, Wahed MA, Fuchs GJ, Baqui AH, Alvarez JO: Simultaneous zinc and vitamin A supplementation in Bangladeshi children: randomised double blind controlled trial. BMJ 2001;323:314318.

53 Khatun UH, Malek MA, Black RE, Sarkar NR, Wahed MA, Fuchs G, Roy SK: A randomized controlled clinical trial of zinc, vitamin A or both in undernourished children with persistent diarrhea in Bangladesh. Acta Paediatr 2001;90:376-380.

-54 Villamor E, Mbise R, Spiegelman D, Hertzmark E, Fataki M, Peterson KE, Ndossi G, Fawzi WW: Vitamin A supplements ameliorate the adverse effect of HIV-1, malaria, and diarrheal infections on child growth. Pediatrics 2002;109:E6.

55 Bhandari N, Bahl R, Saxena M, Taneja S, Bhan MK: Prognostic factors for persistent diarrhoea managed in a community setting. Indian J Pediatr 2000;67:739-745.

56 Valentiner-Branth P, Steinsland H, Santos G, Perch M, Begtrup K, Bhan MK, Dias F, Aaby $\mathrm{P}$, Sommerfelt H, Molbak K: Communitybased controlled trial of dietary management of children with persistent diarrhea: sustained beneficial effect on ponderal and linear growth. Am J Clin Nutr 2001;73:968-974.

57 Jones G, R Steketee RW, Black RE, Bhutta ZA, Morris SS and the Bellagio Child Survival Study Group: How many child deaths can we prevent this year? Lancet 2003;362:65-71.

-58 Travis P, Bennett S, Haines A, Pang T, Bhutta Z A, Hyder AA, Pielemeier NR, Mills A, Evans T: Overcoming health-systems constraints to achieve the Millennium Development Goals. Lancet 2004;364:900-906.

59 Sheth M, Obrah M: Diarrhea prevention through food safety education. Indian J Pediatr 2004; 71:879-882.

60 Curtis V, Cairncross S: Effect of washing hands with soap on diarrhoea risk in the community: a systematic review. Lancet Infect Dis 2003;3: 275-281.

-61 Luby SP, Agboatwalla M, Painter J, Altaf A, Billhimer WL, Hoekstra RM: Effect of intensive handwashing promotion on childhood diarrhea in high-risk communities in Pakistan: a randomized controlled trial. JAMA 2004;291: 2547-2554. 